12

\title{
Мобильный ускоритель на базе безжелезного импульсного бетатрона для радиографирования динамических объектов
}

\author{
(С) Ю.П. Куропаткин, В.И. Нижегородцев, И.Н. Романов, К.В. Савченко, В.Д. Селемир, Е.В. Урлин, \\ B.А. Фомичёв, А.А. Чинин, О.А. Шамро
}

Российский федеральный ядерный центр - Всероссийский научно-исследовательский институт экспериментальной фризики,

607190 Саров, Нижегородская обл., Россия

e-mail: vaalfom@gmail.com

Поступило в Редакцию 27 июня 2021 г.

В окончательной редакции 5 октября 2021 г.

Принята к публикации 14 октября 2021 г.

Дано описание мобильного ускорителя, созданного на базе безжелезного импульсного бетатрона. Ускоритель предназначен для радиографирования динамических объектов с большими оптическими толщинами и позволяет получать до трех $\gamma$-импульсов за один цикл ускорения. Приведено описание работы ускорителя и результаты его тестовых включений в одноимпульсном режиме. Оцененная граничная энергия электронного пучка составила $60 \mathrm{MeV}$ при значении емкости накопителя системы импульсного питания электромагнита бетатрона $1.8 \mathrm{mF}$. Толщина просвеченного свинцового тест-объекта составила $140 \mathrm{~mm}$ на расстоянии $4 \mathrm{~m}$ от источника излучения (мишени бетатрона), длительность выходного $\gamma$-импульса на полувысоте $-120 \mathrm{~ns}$, размеры источника излучения $-3 \times 6 \mathrm{~mm}$. Применение таких ускорителей в составе рентгенографического комплекса позволит оптимизировать схему газодинамических опытов, снизить стоимость последних и, как следствие, повысить эффективность исследований.

Ключевые слова: ускоритель электронов, $\gamma$-импульс, просвечивающая способность, рентгенограмма.

DOI: $10.21883 / J T F .2022 .02 .52021 .196-21$

\section{Введение}

Большой объем экспериментальных задач по исследованию динамических процессов в оптически плотных средах диктует необходимость создания мощных источников импульсного излучения с оптимальным спектральным составом. Информативность подобных исследований помимо интенсивности и спектрального состава излучения определяется также пространственным разрешением, количеством генерируемых импульсов в одном опыте, их длительностью и воспроизводимостью параметров излучения.

Источники, генерирующие излучение для радиографирования динамических объектов, в зависимости от способа ускорения частиц можно условно разделить на линейные [1] и циклические [2,3]. Оба способа имеют свои преимущества и недостатки, а целесообразность их применения определяется конкретной задачей.

На протяжении длительного времени во ФГУП „РФЯЦ-ВНИИЭФ“ и ФГУП „РФЯЦ-ВНИИТФ им. академика Е.И. Забабахина“ для проведения газодинамических исследований (см., например, [4]) применяются рентгенографические комплексы на базе безжелезных импульсных малогабаритных бетатронов (бетатронов типа БИМ) [5].

Функционирующие в настоящее время рентгенографические комплексы представляют собой стационарные сооружения, разработка, создание и эксплуатация которых требует значительных финансовых и временны́х ресурсов. С целью минимизации затрат и оптимизации процесса подготовки и проведения газодинамических опытов во ФГУП „РФЯЦ-ВНИИЭФ“ разработана и реализуется концепция применения мобильных рентгенографических комплексов (МРГК). Комплексы включают мобильные циклические ускорители (МЦУ) [6], взрывозащитную камеру (ВЗК) с объектом исследования, системы коллимации рентгеновского излучения и системы регистрации теневых изображений. С помощью нескольких МЦУ осуществляется просвечивание объекта исследования с разных направлений. Фотография одноракурсного МРГК представлена на рис. 1.

МРГК имеет ряд преимуществ по сравнению со стационарной установкой. Во-первых, оптимизируется геометрия опыта путем изменения положения источников излучения и регистраторов относительно объекта исследования, размещенного в ВЗК. Это позволяет повысить интенсивность рентгеновского излучения в плоскости регистратора как минимум в 2 раза по сравнению с действующими отечественными комплексами [7]. Во-вторых, существенно снижается стоимость рентгенографического комплекса за счет отказа от строительства массивных защитных казематов, тем самым сокращаются затраты на содержание инфраструктуры. В-третьих, упрощается задача создания системы защиты окружающей среды в связи с локализацией опасных продуктов взрыва и вызываемой им ударной волны в пределах ВЗК. В-четвертых, увеличивается календарное время газодинамических испытаний благодаря повы- 


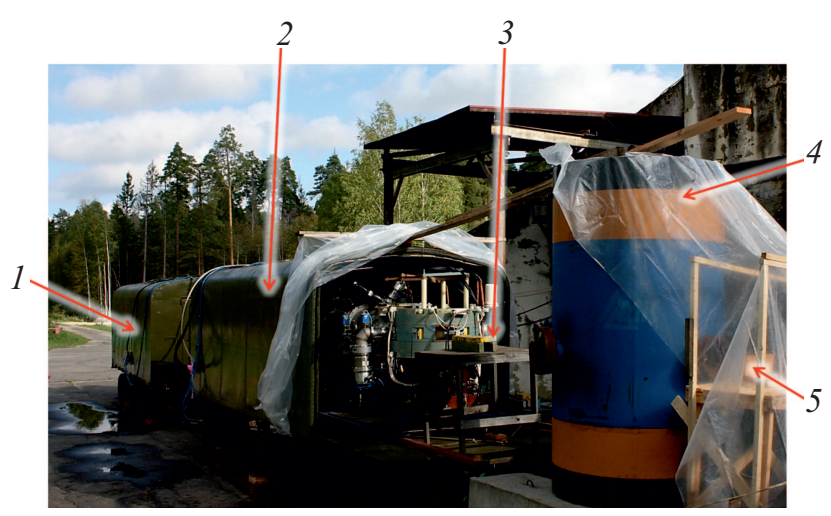

Рис. 1. Фотография одноракурсного мобильного рентгенографического комплекса: 1 - модуль импульсного питания электромагнита бетатрона, 2 - ускорительный модуль, 3 - система коллимации рентгеновских лучей, $4-$ ВЗК, 5 - система регистрации теневых изображений.

шению пожаробезопасности (в настоящее время изза высокой пожароопасности в летний период опыты не проводятся).

В настоящей работе представлены результаты работ, проводимых во ФГУП „РФЯЦ-ВНИИЭФ“ по созданию, конструированию, испытанию и оптимизации ускорителей на базе бетатрона типа БИМ, применяемых в МРГК.

\section{1. Описание мобильного ускорителя}

Основной составляющей МРГК является МЦУ. На рис. 2 представлена фотография одноракурсного одноимпульсного МЦУ на базе бетатрона типа БИМ (МЦУ БИМ), состоящего из двух модулей - ускорительного модуля (УМ) и модуля импульсного питания электромагнита (МИПЭ) бетатрона. Каждый модуль размещен в своем отдельном фургоне.

В фургоне ускорительного модуля 2 располагаются элементы рентгеновской установки, в другом фургоне 1 - система импульсного питания электромагнита бетатрона и технологическое оборудование. Связь между модулями, как и связь с внешними элементами автоматизированной системы управления и контроля, с системой сбора и обработки информации, осуществляется с помощью кабельных и волоконно-оптических линий.

Структурная схема МЦУ БИМ приведена на рис. 3. В состав МЦУ БИМ входят бетатрон, инжектор, система высоковольтного питания, низковольтная и высоковольтная системы синхронизации, автоматизированная система управления и контроля [8], система сбора и обработки информации о выходных параметрах излучателя, система сброса электронного пучка на мишень („медленный“ и „быстрый“ сброс), технологические системы и системы жизнеобеспечения. На рис. 3 изображены линии связи перечисленных систем с модулями инжектора и бетатрона. Соединительные линии с коротким штрихом показывают связь системы сброса

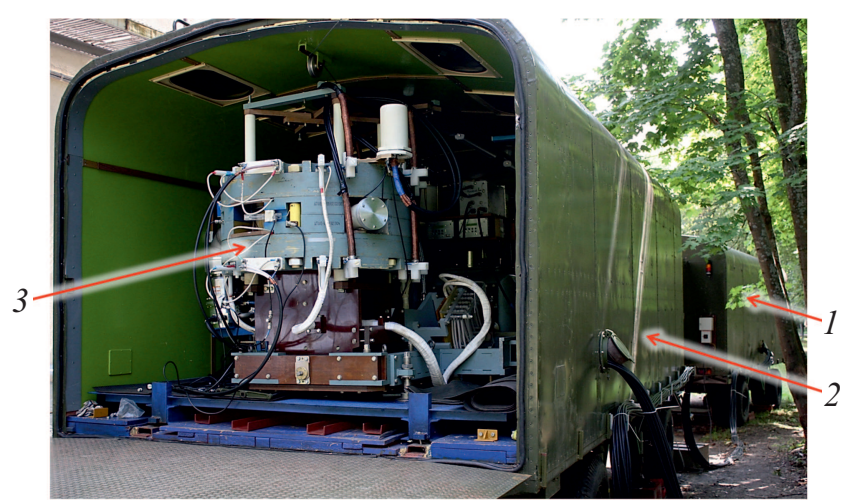

Рис. 2. Фотография одноракурсного трехимпульсного МЦУ: 1 - фургон модуля импульсного питания электромагнита бетатрона, $2-$ фургон ускорительного модуля, 3 - бетатрон типа БИМ.

электронного пучка на мишень с модулями инжектора и бетатрона, линии с длинным штрихом - связь системы высоковольтного питания с модулями инжектора и бетатрона, штрих-пунктирные линии - связь технологических систем и систем жизнеобеспечения с модулями инжектора и бетатрона, линии с длинным штрихом и двойным пунктиром - связь системы синхронизации и автоматизированной системы управления и контроля с модулями инжектора и бетатрона, сплошная линия соответствует связи системы сбора и обработки информации непосредственно с бетатроном.

Базовой установкой МЦУ БИМ является безжелезный импульсный бетатрон нового поколения. Один из основных узлов установки - инжектор, включающий в себя генератор импульсного напряжения (ГИН) [9] и двойную формирующую линию (ДФЛ) [10]. За счет перехода от одинарной формирующей линии (ОФЛ) к ДФЛ удалось уменьшить массогабаритные характеристики источника излучения в 2.5 раза по сравнению с источниками излучения действующих отечественных комплексов [11]. Размеры установки $-4.5 \times 2 \times 1.8 \mathrm{~m}$, общий вес $\approx 5 \mathrm{t}$.

В МИПЭ размещен емкостный накопитель, расчитанный на энергию $\approx 0.5 \mathrm{MJ}$ (6 конденсаторов по $300 \mu \mathrm{F}$, $24 \mathrm{kV}$ ), и высоковольтное оборудование. Размеры емкостного накопителя вместе со стойкой коммутации составляют $2.6 \times 1.4 \times 1.8 \mathrm{~m}$, вес $\approx 2 \mathrm{t}$ (стойка коммутации осуществляет передачу энергии от емкостного накопителя к электромагниту бетатрона).

\section{2. Описание принципа работы МцУ}

Ускорение электронов в бетатроне осуществляется вихревым электрическим полем, возникающем при разряде емкостного накопителя на обмотки электромагнита. В обмотках электромагнита формируется униполярный импульс тока длительностью $1 \mathrm{~ms}$ по основанию. Поскольку тепловые потери в данном случае пренебре- 


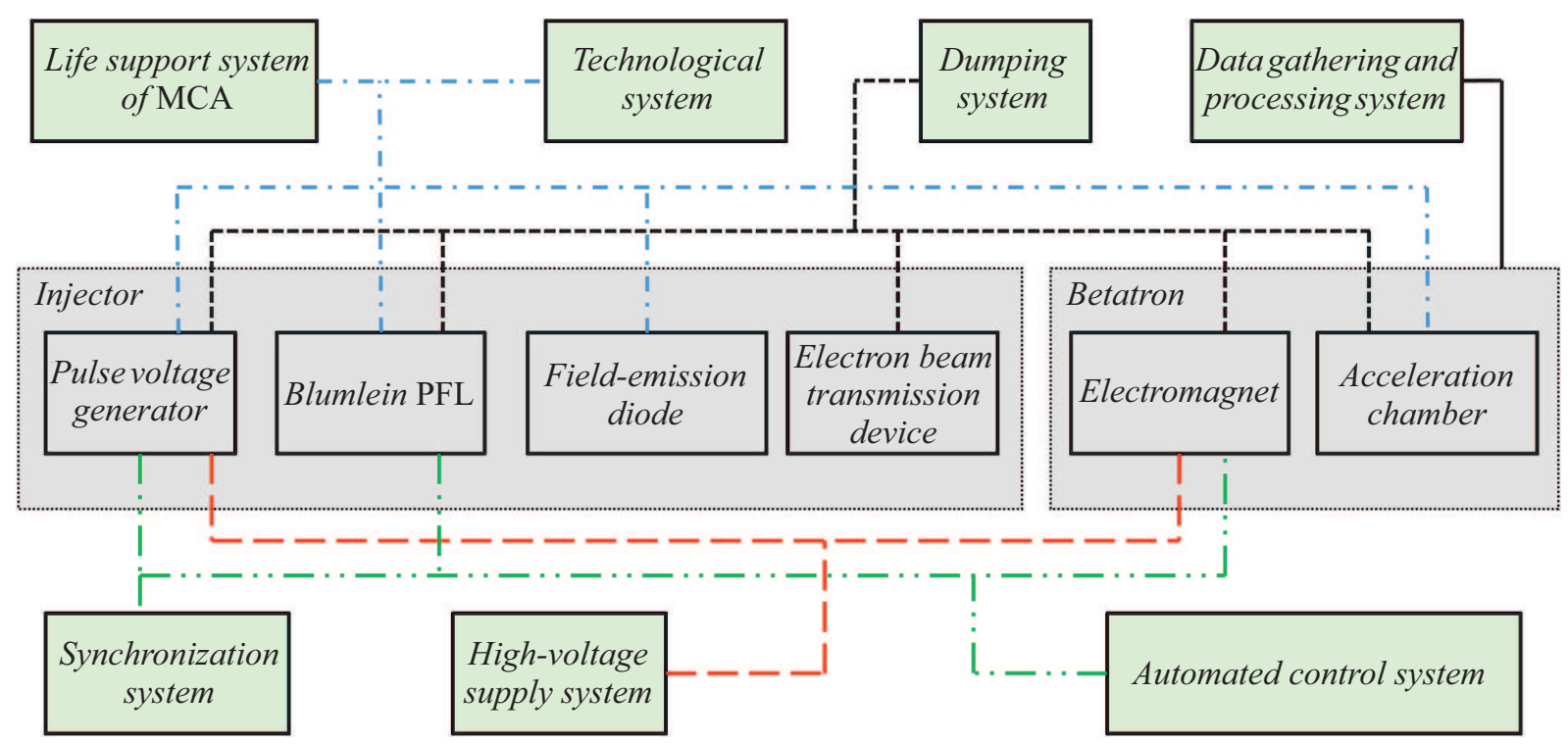

Рис. 3. Структурная схема МЦУ БИМ.

жимо малы, а коэффициент затухания колебаний меньше резонансной частоты контура примерно в 50 раз, то для оценки изменения магнитного поля используется следующее соотношение: $B(t)=B_{0} \sin (\omega t)$, где $\omega=1 /(L C)^{1 / 2}-$ резонансная частота контура $(L$ и $C-$ индуктивность и емкость контура), а $B_{0}=K I_{\max }-$ поле на орбите в максимуме тока электромагнита $I_{\max }$. Здесь $K-$ коэффициент преобразования. Изменение индукции магнитного поля происходит синхронно с ускорением электронов за счет выбора геометрии обмоток электромагнита, что и обеспечивает удержание пучка электронов на орбите.

Высоковольтный блок питания электромагнита включается первым, формируя сигнал „0 поля“, с приходом которого начинается отсчет времени включения систем установки (ГИН, ДФЛ, устройство проводки [12] и т.д.).

Электронный пучок генерируется и предварительно ускоряется в инжекторе. Последний представляет собой ускоритель прямого действия на $1.5 \mathrm{MeV}$. Работает инжектор следующим образом. При срабатывании ГИН осуществляется зарядка ДФЛ. После срабатывания газонаполненного разрядника ДФЛ формирует рабочий импульс напряжения. Далее через промежуточный разрядник этот импульс передается на диод с взрывоэмиссионным катодом, где генерируется пучок электронов с амплитудой тока $\approx 2 \mathrm{kA}$ и длительностью на полувысоте $\approx 10 \mathrm{~ns}$. Инжекция пучка в бетатрон осуществляется с помощью устройства проводки в момент $t_{i n j}$, когда магнитное поле на орбите достигает величины $B_{i n j}$, определяемой соотношениями $B_{i n j}=B_{0} \sin \left(\omega t_{i n j}\right)=E /\left(300 R_{0}\right)$ [13]. Здесь $R_{0}$ - радиус равновесной орбиты (в $\mathrm{m}), E$ - энергия электронного пучка (в $\mathrm{MeV})$. Из последнего равенства может быть найден момент инжектирования.
Проводка и фокусировка электронного пучка осуществляется с помощью 2-х магнитных линз, которые запитываются от высоковольтных блоков питания. Электроны, ускоренные вихревым электрическим полем электромагнита до максимальной энергии, сбрасываются на танталовую мишень за счет возмущения бетатронного поля. Возмущение обеспечивают специальные обмотки сброса, расположенные в электромагните и запитываемые от генераторов медленного и быстрого сброса.

Сброс электронного пучка на мишень происходит в одноимпульсном или в трехимпульсном режиме. В одноимпульсном режиме формируется моноимпульс $\gamma$ излучения при одновременном срабатывании всех трех секций генератора быстрого сброса. Трехимпульсный режим характеризуется формированием трех пиков $\gamma$ излучения. В этом случае используются оба генератора. Сначала срабатывает генератор медленного сброса и орбита с пучком электронов сжимается, приближаясь к мишени. Затем последовательно срабатывают три секции генератора быстрого сброса, формирующие три импульса тока нарастающей амплитуды в обмотках быстрого сброса. Происходит порционный сброс пучка на мишень.

В последние несколько лет проходила отработка МЦУ БИМ с последовательным увеличением емкости конденсаторной батареи системы импульсного питания электромагнита бетатрона и соответствующим увеличением граничной энергии электронного пучка [14]. Во время отработки осуществлялась отладка и оптимизация как отдельных подсистем излучателя, так и ускорителя в целом. Проводился подбор временны́х параметров включения высоковольтных подсистем и величин напряжения импульсного питания для получения максимальной интенсивности выходного излу- 
чения. Исследовалась электромагнитная совместимость оборудования.

\section{3. Результаты тестовых включений МЦу}

В течении последнего года проводилось тестирование установки МЦУ БИМ при значении емкости накопителя системы импульсного питания электромагнита бетатрона $1.8 \mathrm{mF}$. Соответствующая этой емкости граничная энергия электронного пучка была определена по формуле $E=300 B_{0} R_{0}$ путем выполнения оценочного расчета. Радиус равновесной орбиты $R_{0}$ составляет $234 \mathrm{~mm}$. Значение поля $B_{0}$ на этой орбите было найдено в результате моделирования распределения магнитного поля, создаваемого обмотками электромагнита. При измеренном в обмотках электромагнита токе $\approx 80 \mathrm{kA}$ значение поля $B_{0}$ составило $0.85 \mathrm{~T}$. Следовательно, граничная энергия электронного пучка равна $60 \mathrm{MeV}$.

Было проведено порядка 300 включений установки при рабочих зарядных напряжениях. Установка была выведена на рабочие параметры, а полученные осциллограммы свидетельствуют о срабатывании всех систем ускорителя в штатном режиме. На рис. 4 представлена временна́я диаграмма срабатывания подсистем ускорителя при тестовых включениях. Вставка в правом верхнем углу соответствует увеличенному фрагменту диаграммы в окрестности временно́й точки $t_{i n j}=7.3 \mu \mathrm{s}$. Эта точка соответствует времени инжекции электронного пучка в камеру бетатрона в момент максимального тока в обмотках соленоида и магнитной линзы устройства проводки. Циркулирующий в камере бетатрона электронный пучок

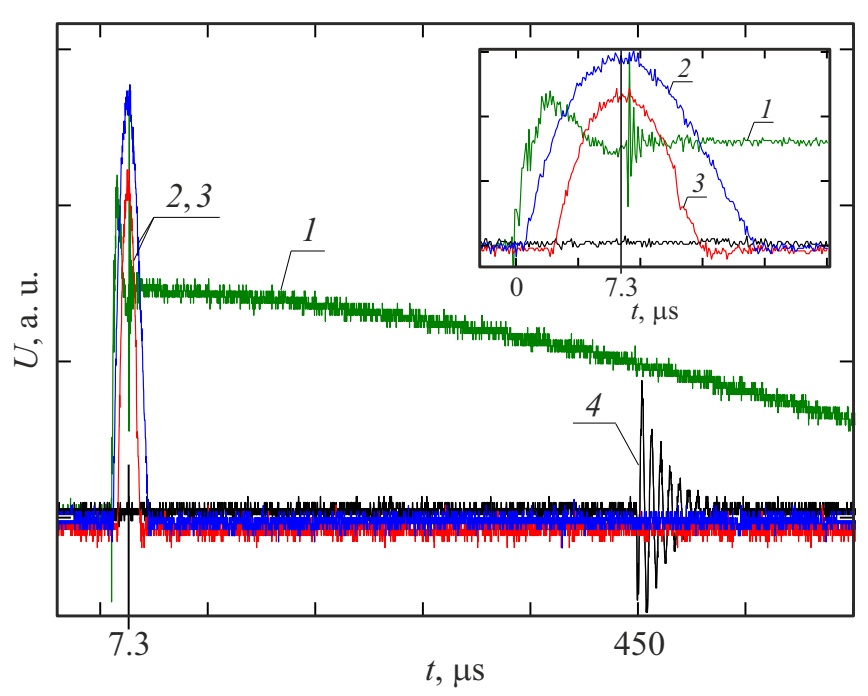

Рис. 4. Временна́я диаграмма срабатывания подсистем ускорителя: 1 - сигнал с датчика „0-поля“ (сигнал при срабатывании электромагнита), 2 - сигнал импульса тока в соленоиде устройства проводки электронного пучка, 3 - сигнал импульса тока в магнитной линзе устройства проводки, 4 - сигнал импульса тока при срабатывании генератора быстрого сброса.

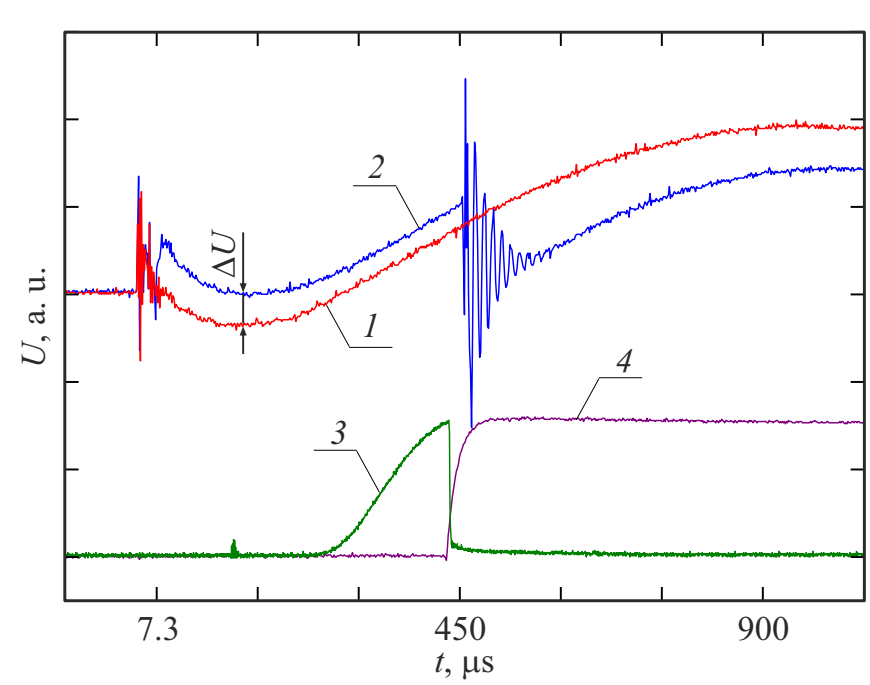

Рис. 5. Осциллограммы сигналов с датчиков МЦУ БИМ: 1 - сигнал с пояса Роговского при отсутствии тока электронов в камере бетатрона, 2 - сигнал с пояса Роговского при наличии тока электронов в камере бетатрона, 3 - сигнал с оптического датчика интенсивности синхротронного излучения, 4 - интенсивность тормозного излучения.

сбрасывается на мишень при $t_{d r o p}=450 \mu$ о относительно сигнала „0-поля“ при срабатывании генератора быстрого сброса.

Типичные осциллограммы сигналов с датчиков контроля выходных параметров приведены на рис. 5. Ток электронного пучка, циркулирующего на орбите бетатрона, измерялся с помощью пояса Роговского, расположенного на внешней поверхности ускорительной камеры ортогонально направлению движения электронного пучка. По осциллограммам (графики 1 и 2 на рис. 5) измерялась разница сигналов $\Delta U$ с пояса Роговского при отсутствии и наличии тока электронов в камере [15]. Первая осциллограмма представляет собой реперную линию, которая получается при срабатывании электромагнита бетатрона без инжекции электронов в ускорительную камеру, а вторая - рабочий импульс. Пересчет разницы $\Delta U$ в ток электронного пучка в камере бетатрона проводился с помощью калибровочной процедуры, использующей дополнительную катушку, намотанную на пояс Роговского, и специально разработанное устройство, включающее в себя токовый шунт с известным сопротивлением, конденсатор и трехпозиционный переключатель. Как видно из графика 2, на 450-ой $\mu \mathrm{s}$ появляются высокочастотные колебания, связанные с наводкой при срабатывании генератора быстрого сброса электронного пучка на мишень. График 3 на рис. 5 представляет собой сигнал с оптического датчика интенсивности синхротронного излучения электронного пучка в камере бетатрона. График 4 на рис. 5 - сигнал с датчика интенсивности тормозного излучения (датчик типа СППД, включенный в режиме интегрирования). Все представленные осциллограммы соответствуют од- 

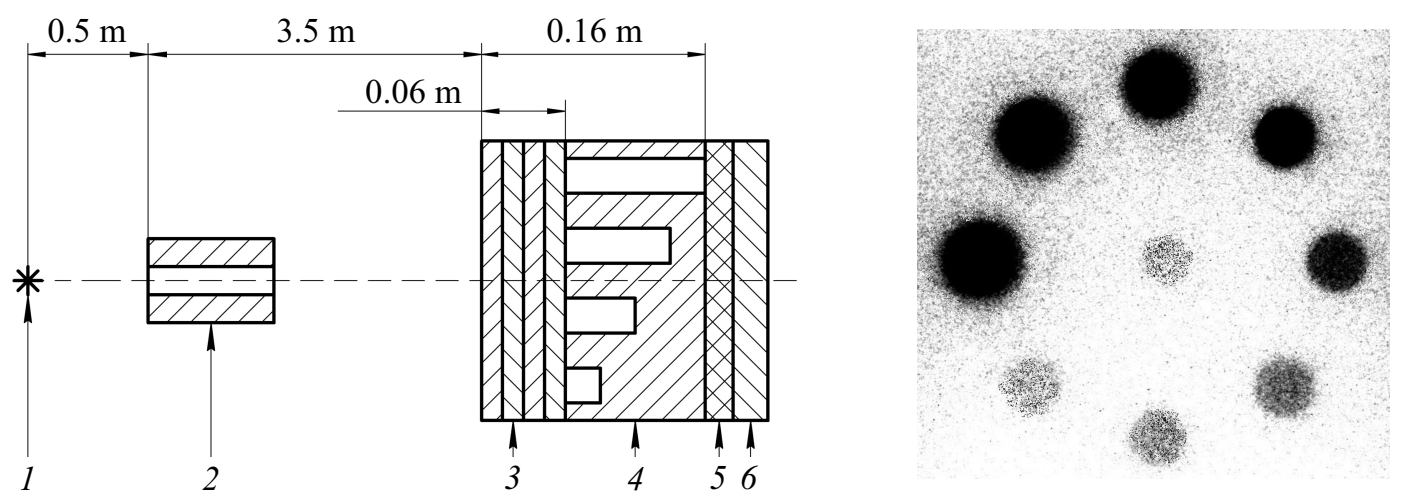

Рис. 6. Радиографирование тест-объекта для определения просвечивающей способности установки МЦУ БИМ. $a-$ схема эксперимента: 1 - источник излучения, $2-$ система коллимации рентгеновских лучей, 3 - свинцовые пластины, $4-$ свинцовый тест-объект, 5 - кассета ImagePlate, 6 - свинцовый защитный экран; $b$ - рентгенограмма тест-объекта.

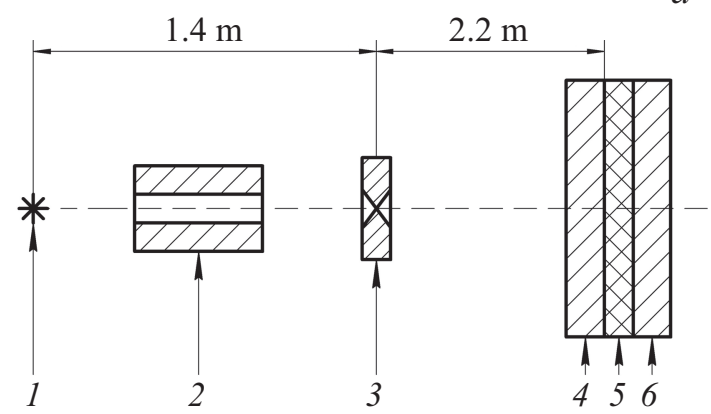

$b$

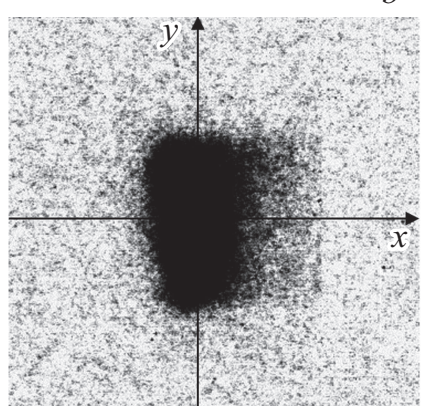

西

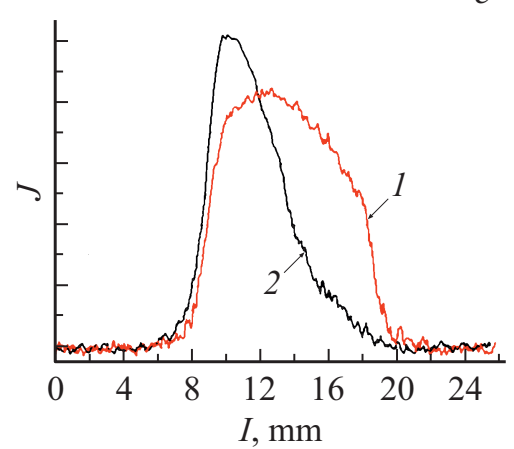

Рис. 7. Определение размеров источника рентгеновского излучения. $a-$ схема эксперимента: $1-$ источник излучения, $2-$ система коллимации рентгеновских лучей, 3 - камера-обскура, 4 - свинцовый фильтр, 5 - кассета ImagePlate, 6 - свинцовый защитный экран; $b$ - рентгенограмма источника излучения; $c$ - денситограмма изображения источника рентгеновского излучения: график 1 соответствует плотности почернения изображения вдоль оси $x$, график 2 соответствует плотности почернения изображения вдоль оси $y$.

ному включению, зарядные напряжения емкостных накопителей систем излучателя соответствуют штатным значениям.

Для оценки просвечивающей способности излучателя было проведено радиографирование тест-объекта, представляющего собой свинцовый параллелепипед с углублениями. Перед тест-объектом располагались четыре свинцовые пластины. Минимальная толщина свинца составляла $60 \mathrm{~mm}$ с последующим нарастанием толщины через $10 \mathrm{~mm}$ в тест-объекте. На рис. 6, $а$ приведена схема данного эксперимента. Регистрация теневого изображения тест-объекта и источника излучения проводилась с помощью кассеты ImagePlate. Рентгенограмма тестобъекта приведена на рис. $6, b$. Максимальная толщина просвеченного вместе с пластинами тест-объекта на расстоянии $4 \mathrm{~m}$ от танталовой мишени бетатрона составила $140 \mathrm{~mm}$.

Для оценки размеров источника тормозного излучения применялась камера-обскура. На рис. 7, $a$ представлена схема эксперимента по определению размеров источника излучения. Рентгенограмма источника излучения и ее денситограмма приведены соответственно на рис. $7, b$ и $c$. Анализ полученных результатов показал: в геометрии тестовых включений с учетом геометрического коэффициента $k=l_{2} / l_{1}=1.6$ (здесь $l_{1}$ - расстояние от источника излучения до камеры-обскуры, $l_{2}-$ расстояние от камеры-обскуры до кассеты ImagePlate) размеры источника излучения составляют $3 \times 6 \mathrm{~mm}$ (на полувысоте графиков денситограммы) при размерах танталовой мишени $6 \times 6 \mathrm{~mm}$. Следует отметить, что конструкция камеры бетатрона позволяет в зависимости от задач эксперимента варьировать размеры танталовой мишени (как показали ранее проведенные исследования, уменьшение размеров мишени в два раза ведет к уменьшению интенсивности выходного тормозного излучения на $15-20 \%$ ).

Для оценки длительности импульса тормозного излучения использовался сцинтилляционный детектор с конвертером излучения на основе стильбена [16]. Типичная осциллограмма сигнала $\gamma$-импульса с детектора пред- 


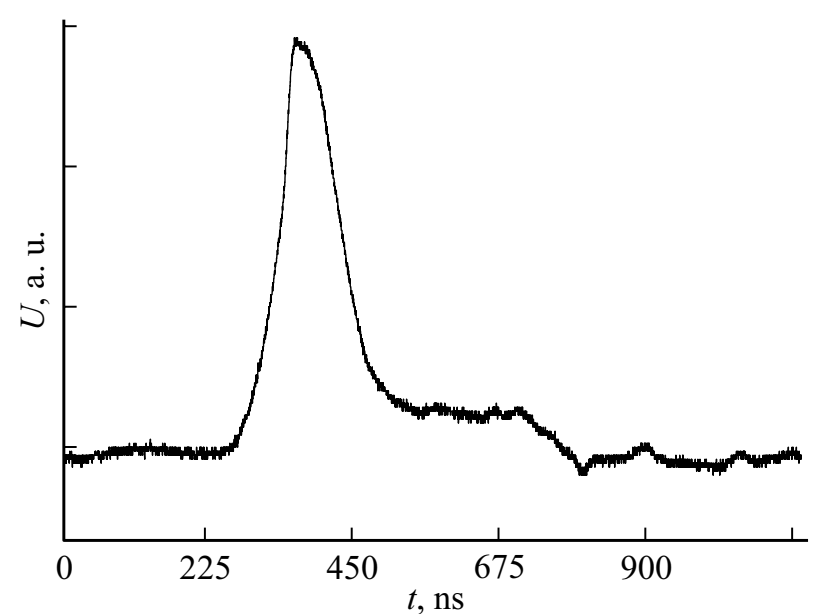

Рис. 8. Осциллограмма сигнала $\gamma$-импульса со сцинтилляционного датчика.

ставлена на рис. 8. Определенная по осциллограммам длительность выходного $\gamma$-импульса в одноимпульсном режиме на полувысоте составляет $\approx 120 \mathrm{~ns}$.

\section{Заключение}

В результате многолетней работы, проводимой во ФГУП „РФЯЦ-ВНИИЭФ“, был создан, испытан и оптимизирован мобильный циклический ускоритель на базе бетатрона типа БИМ (МЦУ БИМ). Тестовые включения МЦУ БИМ показали, что при оцененной граничной энергии электронного пучка $E=60 \mathrm{MeV}$ толщина просвеченного свинцового тест-объекта на расстоянии $4 \mathrm{~m}$ от танталовой мишени с размерами $6 \times 6 \mathrm{~mm}$ составила $140 \mathrm{~mm}$, длительность выходного $\gamma$-импульса на полувысоте в одноимпульсном режиме - $120 \mathrm{~ns}$, размеры источника излучения в геометрии тестовых включений $3 \times 6 \mathrm{~mm}$.

В дальнейшем планируется продолжить тестирование МЦУ БИМ и вывести излучатель на рабочие параметры в трехимпульсном режиме. Кроме того, необходимо осуществить работы по созданию модуля управления и контроля, расположенного в отдельном фургоне и включающего в себя автоматизированную систему управления и контроля, систему сбора и обработки информации, а также рабочие места для операторов и оперативноремонтного персонала. В этом случае будет полностью реализован автономный мобильный циклический ускоритель для радиографирования динамических объектов с большими оптическими толщинами.

\section{Благодарности}

Авторы выражают искреннюю благодарность Г.В. Борискову и А.А. Тренькину за полезные советы и критические замечания, высказанные при подготовке рукописи.

\section{Конфликт интересов}

Авторы заявляют, что у них нет конфликта интересов.

\section{Список литературы}

[1] K. Peach, C. Ekdahl. In. Accelerators for high intensity beams, ed. by A.W. Chao, W. Chou (World Scientific, Singapore, 2013), p. 126-129.

[2] В.А. Москалев, В.Л. Чахлов. Бетатроны (Изд-во Томского политех. уни-та, Томск, 2009)

[3] А.Р. Ахметов, А.А. Каргин, П.А. Колесников, О.А. Никитин, С.Д. Хренков. Сборник материалов ХІІ Междунар. конф. „Забабахинские научные чтения“ (Снежинск, Россия, 2014), с. 50-52.

[4] N.I. Egorov, G.V. Boriskov, A.I. Bykov, Yu.P. Kuropatkin, N.B. Lukynov, V.D. Mironenko, V.N. Pavlov. Contributions Plasma Physics, 51, 333-338 (2011).

[5] В.А. Комрачков, К.Н. Панов, А.М. Подурец. В сб.: Невозмущающие методы диагностики быстропротекающих процессов, под ред. А.Л. Михайлова („РФЯЦ-ВНИИЭФ“, Саров, 2015), с. 112-114.

[6] Д.И. Зенков, Ю.П. Куропаткин, В.И. Нижегородцев, В.Д. Селемир, О.А. Шамро. Пат. 2548585 РФ: C1 RU МПК G03B 42/02, заявитель и патентообладатель ГК „Росатом“, 2015, БИ № 11.

[7] И.И. Винтизенко. Линейные индукционные ускорители (Физматлит, М., 2016), с. 21.

[8] O.V. Belov, R.V. Valekzhanin, D.V. Kustov, O.A. Shamro, T.V. Sharov. IOP Conf. Series: Mater. Sci. Engineer. (China, 2017), v. 199, p. 012048.

[9] В.С. Босамыкин, А.И. Герасимов, А.И. Павловский. ПТЭ, 2, 5 (1997).

[10] R.B. Miller. An Introduction to the Physics of Intense Charged Particle Beams (Plenum Press, NY., London, 1982).

[11] C. Ekdahl. IEEE Trans. Plasma Sci., 30 (1), 254 (2002). DOI: 10.1109/TPS.2002.1003868

[12] G.J. Caporaso, Yu-J. Chen. In. Induction Accelerators, ed. by K. Takayama, R.J. Briggs (Springer, Heidelberg-Dordrecht-London-NY., 2011), p. 125.

[13] А.А. Коломенский, А.Н. Лебедев. Теория циклических ускорителей (Физматгиз, М., 1962), с. 26.

[14] V.A. Fomichev, A.A. Chinin, S.G. Kozlov, Yu.P. Kuropatkin, V.I. Nizhegorodtsev, I.N. Romanov, K.V. Savchenko, V.D. Selemir, O.A. Shamro, E.V. Urlin. 10th Int. Particle Accelerator Conf. (Melbourne, Australia, 2019) DOI: 10.18429/JACoW-IPAC2019-THPMP026

[15] А.К. Гренадеров. ПТЭ, 5, 55 (1989).

[16] S.K. Lee, Y.H. Cho, B.H. Kang, W.G. Lee, J.K. Kim, G.D. Kim, N.Z. Galunov, Y.K. Kim. Progress in Nuclear Science and Technology, 1, 292-295 (2011). 